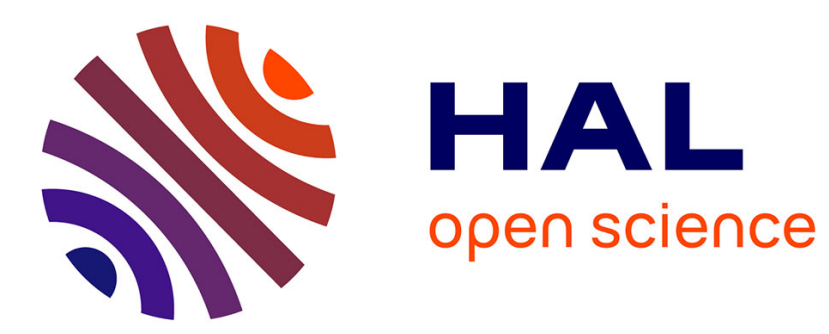

\title{
Artificial boundary conditions for the semi-discretized one-dimensional nonlocal Schrödinger equation
}

\author{
Songsong Ji, Gang Pang, Xavier Antoine, Jiwei Zhang
}

\section{To cite this version:}

Songsong Ji, Gang Pang, Xavier Antoine, Jiwei Zhang. Artificial boundary conditions for the semidiscretized one-dimensional nonlocal Schrödinger equation. Journal of Computational Physics, 2021, 444, pp.110575. 10.1016/j.jcp.2021.110575 . hal-02898080

\section{HAL Id: hal-02898080 \\ https://hal.science/hal-02898080}

Submitted on 13 Jul 2020

HAL is a multi-disciplinary open access archive for the deposit and dissemination of scientific research documents, whether they are published or not. The documents may come from teaching and research institutions in France or abroad, or from public or private research centers.
L'archive ouverte pluridisciplinaire $\mathbf{H A L}$, est destinée au dépôt et à la diffusion de documents scientifiques de niveau recherche, publiés ou non, émanant des établissements d'enseignement et de recherche français ou étrangers, des laboratoires publics ou privés. 


\title{
Artificial boundary conditions for the semi-discretized one-dimensional nonlocal Schrödinger equation
}

\author{
Songsong $\mathrm{JI}^{\mathrm{a}}$, Gang PANG ${ }^{\mathrm{b}, *}$, Xavier ANTOINE ${ }^{\mathrm{c}}$, Jiwei ZHANG ${ }^{\mathrm{d}}$ \\ ${ }^{a}$ HEDPS,CAPT, and LTCS, College of Engineering, Peking University, Beijing 100871, China \\ ${ }^{b}$ School of Mathematical Science, Beijing University of Aeronautics and Astronautics, Beijing 100083, China \\ ${ }^{c}$ Université de Lorraine, CNRS, Inria, IECL, F-54506 Vandoeuvre-lès-Nancy Cedex, France \\ ${ }^{d}$ School of Mathematics and Statistics, and Hubei Key Laboratory of Computational Science, Wuhan University, \\ Wuhan 430072, China
}

\begin{abstract}
A general method is proposed to build exact artificial boundary conditions for the one-dimensional nonlocal Schrödinger equation. To this end, we first consider the spatial semi-discretization of the nonlocal equation, and then develop an accurate numerical method for computing the Green's function of the semi-discrete nonlocal Schrödinger equation. These Green's functions are next used to build the exact boundary conditions corresponding to the semi-discrete model. Numerical results illustrate the accuracy of the boundary conditions. The methodology can also be applied to other nonlocal models and could be extended to higher dimensions.
\end{abstract}

Keywords: nonlocal Schrödinger equation; semi-discrete scheme; transparent boundary condition; artificial boundary condition

\section{Contents}

\begin{tabular}{llr}
\hline 1 & Introduction & 1
\end{tabular}

2 Exact boundary conditions for the semi-discretized nonlocal Schrödinger equation 3

2.1 Semi-discretization of the 1D nonlocal Schrödinger equation . . . . . . . . . . . 3

2.2 Exact boundary condition for the semi-discrete nonlocal Schrödinger equation. . . . . 4

\begin{tabular}{|llr}
\hline 3 & Full discretization scheme and implementation aspects & 8
\end{tabular}

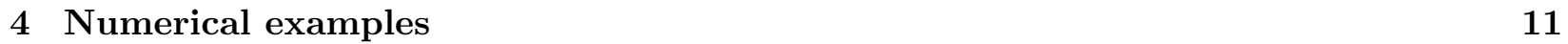

4.1 Example 1. . . . . . . . . . . . . . . . . . . . . . . . . . . . . . 11

4.2 Example 2 . . . . . . . . . . . . . . . . . . . . . . . . . . . . . . . . . . 12

4.3 Example 3 . . . . . . . . . . . . . . . . . . . . . . . . . . . . . . . . . . 12

$\begin{array}{llr}5 \text { Conclusion } & 14\end{array}$

\section{Introduction}

The time-dependent Schrödinger equation is widely used in the modeling of physical phenomena, with many applications in quantum mechanics, optics, acoustics or electromagnetism for instance

*Author for correspondence, xavier.antoine@univ-lorraine.fr 
(see e.g. [1, 2, 3]). More precisely, in the one-dimensional case, the simplest equation writes: find the wave function $\psi$ solution to

$$
\left\{\begin{array}{l}
\mathrm{i} \psi_{t}(x, t)=-\partial_{x}^{2} \psi(x, t)+V(x) \psi(x, t), \quad x \in \mathbb{R}, \quad t \in(0, T] \\
\psi(x, 0)=\psi^{0}(x), \quad x \in \mathbb{R} \\
\lim _{|x| \rightarrow+\infty} \psi(x, t)=0, \quad t \in(0, T]
\end{array}\right.
$$

where $\mathrm{i}=\sqrt{-1}, V$ is a smooth real-valued potential and $\psi^{0}$ is a given initial data that we assume to be compactly supported. The maximal time of computation is denoted by $T$. Numerically solving system (1) is now classical by using many possible schemes [4], both in space and time according to the configuration. In particular, one of the well-known difficulties related to (1) concerns the problem of the suitable truncation of the infinite spatial domain. Many contributions are available in the literature to fix this problem, most specifically by using transparent/artificial/absorbing boundary conditions (ABC) at a fictitious boundary, or alternatively absorbing layers or Perfectly Matched Layer (PML). Extensions to more general situations can also be found, like for example for higher dimensions, unbounded space and time dependent potentials, nonlinear terms among others. We refer e.g. to [5, 6] for more details about this important topic for quantum mechanics equations.

Two decades ago, Laskin [7, 8, 9, 10] introduced a Fractional Schrödinger Equation (FSE) which was used to describe for example the fractional oscillator of Bohr atom [7] or long-range dispersive interactions [11]. Basically, the equation involves a fractional laplacian term $\left(-\partial_{x}^{2}\right)^{s / 2}$ in (1), usually for $1 \leq s \leq 2$ ( $s=2$ corresponding to the standard local situation). Since then, the FSE has been studied intensively from both the applicative, theoretical and numerical points of view (see e.g. 12, 13, 14, 15, 16, 17, 18, 19, 20, 21, 22]), exhibiting specific behaviors compared with the local Schrödinger equation. Even if the physical framework is totally different, let us also remark that closely related mathematical forms of such problems arise for the fractional heat equation [23] which is useful in many situations. More generally, fractional PDE models can be included into the wider framework of nonlocal PDEs [24] which allows to consider e.g. more advanced interactions at distance not available in the fractional situation. Considering this point of view, the fractional PDE model corresponds to a specific kernel for the nonlocal model. In the present paper, we focus on the one-dimensional initial boundary-value problem with nonlocal Schrödinger equation, which generalizes then the FSE: find the function $\psi$ solution to

$$
\left\{\begin{array}{l}
\mathrm{i} \psi_{t}(x, t)=\mathcal{L}_{\eta} \psi(x, t)+V(x) \psi(x, t), \quad x \in \mathbb{R}, \quad t \in(0, T], \\
\psi(x, 0)=\psi^{0}(x), \quad x \in \mathbb{R} \\
\lim _{|x| \rightarrow+\infty} \psi(x, t)=0, \quad t \in(0, T] .
\end{array}\right.
$$

The nonlocal operator $\mathcal{L}_{\delta}$ appearing in (2) which replaces the local laplacian term in (1) can be defined under the integral form

$$
\mathcal{L}_{\eta} \psi(x)=\int_{\mathbb{R}} \gamma_{\eta}\left(y-x, \frac{y+x}{2}\right)(\psi(x)-\psi(y)) d y .
$$

The interaction kernel function $\gamma_{\eta}$ has the following properties

- positiveness: $\gamma_{\eta}(\alpha, \beta) \geq 0$;

- symmetry according to the first argument $\alpha$ : $\gamma_{\eta}(-\alpha, \beta)=\gamma_{\eta}(\alpha, \beta)$;

- finite horizon: $\exists \eta>0$ such that $\gamma_{\eta}(\alpha, \beta)=0$ if $|\alpha|>\eta>0$. 
The nonlocal operator $\mathcal{L}_{\eta}$ is known to have some connections with differential operators. Indeed, If one assumes that $\gamma_{\eta}$ is such that the following moment condition holds

$$
0<\sigma(x)=\frac{1}{2} \int_{\mathbb{R}} u^{2} \gamma_{\eta}\left(u, x+\frac{u}{2}\right) d u<\infty,
$$

the nonlocal operator $\mathcal{L}_{\eta}$ then converges to the second-order differential operator

$$
\lim _{\eta \rightarrow 0} \mathcal{L}_{\eta} \psi(x)=-\partial_{x}\left(\sigma(x) \partial_{x}\right) \psi(x)=\mathcal{L}_{0} \psi(x) .
$$

Approximation of the one-dimensional fractional laplacian can also be built thanks to a well-suited kernel $\gamma_{\eta}$ as $\eta \rightarrow 0$. Again, a very similar problem as (2) could also arise for nonlocal diffusion [23, 24].

Developing $\mathrm{ABC}$ or absorbing layers/PML truncation techniques for spatially nonlocal and fractional models is much less studied in the literature than for local PDEs. Concerning ABCs for the nonlocal heat equations, DtN-based operators were introduced recently in [25, 26]. In addition, a thorough numerical analysis of $\mathrm{ABCs}$ has been developed in 27]. ABCs for the two-dimensional nonlocal wave equation were built in [28, 29]. Concerning nonlocal Schrödinger equations, ABCs have been proposed and numerically approximated in [30, 31]. For the absorbing layers/PML approach, efficient and accurate methods have been developed in [32, 33, 34, 35] for the timedependent heat and Schrödinger equations involving fractional operators, in particular the fractional laplacian. It is however not clear whether the approaches extend or not to more general nonlocal models as the one considered in the present paper.

To deal with the nonlocal Schrödinger equation (2), we first propose to use the Asymptotic Compatibility (AC) spatial discretization of the associated operator on a uniform grid. Inspired by previous works in lattice dynamics [36], an efficient algorithm is then designed to accurately compute the Green's function of the semi-discretized nonlocal Schrödinger equation with a single-source. A similar idea has been used to obtain exact ABCs [30, 31] by using the spatial Laplace, $z$-transforms and an iterative technique for the continuous and discrete nonlocal Schrödinger equations, respectively. One difficulty for nonlocal problems is to calculate its kernel function accurately since the numerical Fourier transform and contour integrals would require too much calculations and lead in deviations in large time [30, 31, 37]. Here, we follow the idea in [36, 38, where the kernel functions of the semi-discrete heat and Schrödinger equations on a two-dimensional domain are computed with high accuracy. We derive the recursive relation between the kernel functions such that they can be obtained through an ordinary integro-differential system rather than based on the numerical Fourier transform. As a consequence, the kernel functions can be evaluated with high precision. Based on this computation, we are able to use the kernel functions with a single-source to design exact boundary conditions for the semi-discretized nonlocal Schrödinger equation (2) for the AC scheme. The method can be extended to more general nonlocal semi-discrete systems and should be able to handle higher dimensional problems.

The plan of the paper is the following. Section 2 develops the methodology to obtain the accurate representation of the proposed method, as well as some recursive formulas that will be used for the numerical purpose for the semi-discrete Green's functions. Section 3 considers the full discretization of the equations, and some computational aspects. In Section 4 , we apply the method to three cases to illustrate numerically the properties of the scheme. Finally, Section 5 concludes.

\section{Exact boundary conditions for the semi-discretized nonlocal Schrödinger equation}

\subsection{Semi-discretization of the $1 D$ nonlocal Schrödinger equation}

To design some exact boundary conditions for the nonlocal Schrödinger equation appearing in system (2), we first semi-discretize the equation and next extract the corresponding boundary 
condition. In [39, 40], the authors proved that the Asymptotic Compatibility (AC) discretization ensures that the numerical solution of the nonlocal model converges to the correct continuous solution when $\eta \rightarrow 0$. We use the $\mathrm{AC}$ discretization scheme derived in [28] to approximate the nonlocal operator $\mathcal{L}_{\eta}$. Let us introduce $\phi_{j}$ as the standard hat function of width $\Delta x$ centered at point $x_{j}:=j \Delta x$, where $\Delta x$ is the uniform spatial discretization step. Then, we have the following approximation

$$
L_{\eta, \Delta x} \psi\left(x_{j}\right)=\sum_{k \in \mathbb{Z}} b_{j, k}\left(\psi\left(x_{j}\right)-\psi\left(x_{k}\right)\right)
$$

where the real-valued coefficients $b_{j, k}$ are given by

$$
b_{j, k}= \begin{cases}\frac{1}{(j-k) \Delta x} \int_{\mathbb{R}} y \phi_{j-k}(y) \gamma_{\eta}\left(y, \frac{x_{j}+x_{k}}{2}\right) d y, & j \neq k, \\ 0, & j=k\end{cases}
$$

and $b_{k, j}=b_{j, k}$. Since the kernel $\gamma_{\eta}$ is compactly supported, then one gets: $b_{j, k}=0$ for $|j-k|>$ $K:=[\eta / \Delta x]+1$.

In the case of a one-dimensional laplacian operator $\partial_{x}^{2}$, we can use e.g. one of the following finite-difference schemes

$$
\begin{aligned}
& \psi_{x x}\left(x_{k}\right)=\frac{\psi\left(x_{k-1}\right)-2 \psi\left(x_{k}\right)+\psi\left(x_{k+1}\right)}{(\Delta x)^{2}}+\mathcal{O}\left((\Delta x)^{2}\right), \\
& \psi_{x x}\left(x_{k}\right)=\frac{-\psi\left(x_{k-2}\right)+16 \psi\left(x_{k-1}\right)-30 \psi\left(x_{k}\right)+16 \psi\left(x_{k+1}\right)-\psi\left(x_{k+2}\right)}{(\Delta x)^{4}}+\mathcal{O}\left((\Delta x)^{4}\right) .
\end{aligned}
$$

Let us remark that these formulas can also be written into the form of the discrete nonlocal operator (6), providing then a general framework for our derivation. For $V=0$, we obtain the semi-discrete nonlocal Schrödinger equation approximating (2) as the time-dependent system

$$
\mathrm{i} \dot{\psi}_{j}=a_{0} \psi_{j}+\sum_{k=1}^{K} a_{k}\left(\psi_{j+k}+\psi_{j-k}\right)
$$

with $a_{0}=-2\left(\sum_{k=1}^{K} a_{k}\right)(K \geq 1)$, and, for $1 \leq k \leq K, a_{k} \in \mathbb{R}$. The notation $\dot{\psi}_{j}$ designates the time derivative of the time-dependent function $\psi_{j}$. The extension to the case of a potential $V$ which is constant outside the computational domain is direct.

\subsection{Exact boundary condition for the semi-discrete nonlocal Schrödinger equation}

Let us now consider $(9)$ with a semi-discrete compact initial data $\psi_{j}^{0}$, for $1 \leq j \leq J$. We need to know the values of $\psi_{j}(t)$ from $j=-(K-1)$ to $j=0$ outside the computational domain to close $(9)$ on the left boundary. Similarly, the values of $\psi_{j}(t)$ are required at the right region, for $J+1 \leq j \leq J+K$.

To this end, let us consider the semi-discrete problem with given Dirac source term $\delta(t)$ located at $x_{0}$

$$
\left\{\begin{array}{l}
\mathrm{i} \dot{f}_{j}=a_{0} f_{j}+\sum_{k=1}^{K} a_{k}\left(f_{j+k}+f_{j-k}\right), \quad \text { for } j \neq 0, \\
f_{j}(0)=0, \quad j \neq 0 \\
f_{0}(t)=\delta(t), t>0
\end{array}\right.
$$


The values of $f_{j}(t)(j \neq 0)$ are determined by $\delta(t)$. In addition, it is clear that $f_{j}(t)=f_{-j}(t)$. For $K$ given functions $F_{j}(t)$, with $1 \leq j \leq K$, we assume that there exist $K$ time-dependent functions $G_{k}(t)$, with $1 \leq k \leq K$, such that

$$
F_{j}(t)=\sum_{k=1}^{K}\left(f_{j-k} * G_{k}\right)(t),
$$

where $*$ designates the time convolution product defined by

$$
(f * g)(t):=\int_{0}^{t} g(t-\tau) f(\tau) d \tau .
$$

If we set

$$
\psi_{j}(t)=\sum_{k=1}^{K}\left(f_{j-k} * G_{k}\right)(t),
$$

then we have : $\psi_{j}(t)=F_{j}(t)$, for $1 \leq j \leq K$. On the other hand, for $j \leq 0$ or $j \geq K+1$, the function $\psi_{j}(t)$ satisfies

$$
\begin{aligned}
\mathrm{i} \dot{\psi}_{j}(t) & =\mathrm{i} \sum_{k=1}^{K}\left(\dot{f}_{j-k} * G_{k}\right)(t) \\
& =\sum_{k=1}^{K} a_{0}\left(f_{j-k} * G_{k}\right)(t)+\sum_{j=1}^{K} \sum_{\ell=1}^{K} a_{k}\left(\left(f_{j-k-\ell}+f_{j-k+\ell}\right) * G_{k}\right)(t) \\
& =a_{0} \sum_{k=1}^{K}\left(f_{j-k} * G_{k}\right)(t)+\sum_{\ell=1}^{K} a_{\ell}\left(\sum_{k=1}^{K}\left(f_{j-k-\ell} * G_{k}\right)(t)+\sum_{k=1}^{K}\left(f_{j-k+\ell} * G_{k}\right)(t)\right) \\
& =a_{0} \psi_{j}(t)+\sum_{\ell=1}^{K} a_{\ell}\left(\psi_{j-\ell}(t)+\psi_{j+\ell}(t)\right) .
\end{aligned}
$$

Therefore, we obtain

$$
\left\{\begin{array}{l}
\mathrm{i} \dot{\psi}_{j}=a_{0} \psi_{j}+\sum_{k=1}^{K} a_{k}\left(\psi_{j+k}+\psi_{j-k}\right), \text { for } j \leq 0 \text { and } j \geq K+1, \\
\psi_{j}(0)=0, \quad \text { for } j \leq 0 \text { and } j \geq K+1 \\
\psi_{j}(t)=F_{j}(t), \quad 1 \leq j \leq K
\end{array}\right.
$$

This means that the functions $\psi_{j}(t)$, for $j \leq 0$, satisfy the governing equations of the semi-discrete nonlocal Schrödinger equation with $K$ source terms $F_{j}(t)$, for $1 \leq j \leq K$. Then, we have

$$
\psi_{j}(t)=\sum_{k=1}^{K}\left(f_{j-k} * G_{k}\right)(t), \quad 1 \leq j \leq K,
$$

with

$$
F_{j}(t)=\sum_{k=1}^{K}\left(f_{j-k} * G_{k}\right)(t) .
$$

Let us introduce the $K \times K$ matrix $\mathbb{A}(t)$ such that its $(j, k)$-th element is equal to $f_{j-k}(t)$, and the two vectors $\mathbf{F}(t)=\left[F_{1}, F_{2}, F_{3}, \ldots, F_{K}\right]^{T}$ and $\mathbf{G}(t)=\left[G_{1}, G_{2}, G_{3}, \ldots, G_{K}\right]^{T}$. Then, we obtain: $\mathbf{F}=\mathbb{A} * \mathbf{G}$, 
which leads to $\tilde{\mathbf{G}}=\widetilde{\mathbb{A}}^{-1} \tilde{\mathbf{F}}$, where $\tilde{u}(s)$ designates the time Laplace transform of a function $u(t)$. Let us introduce now the vector $\boldsymbol{\psi}(t)=\left[\psi_{0}, \psi_{-1}, \ldots \psi_{-(K-1)}\right]^{T}$ and the $K \times K$ matrix $\mathbb{B}(t)$, with elements $\mathbb{B}_{j, k}=f_{j+k-1}(t), j=1, \ldots, K, k=0, \ldots,-(K-1)$. From $(14)$, we have $: \widetilde{\boldsymbol{\psi}}=\widetilde{\mathbb{C}} \tilde{\mathbf{F}}$, where $\widetilde{\mathbb{C}}=\widetilde{\mathbb{B}} \widetilde{\mathbb{A}}^{-1}$, yielding

$$
\psi_{1-j}(t)=\sum_{k=1}^{K}\left(\mathbb{C}_{j, k} * F_{k}\right)(t), \quad 1 \leq j \leq K .
$$

The functions $\psi_{j}(t)(j \leq 0)$ outside the computational domain satisfy the governing semi-discrete equations with $K$ sources $\psi_{k}(t)$, for $1 \leq k \leq K$. Therefore, each $\psi_{j}$, for $-(K-1) \leq j \leq 0$, can be represented by $\psi_{k}(t)(1 \leq k \leq K)$ in the computational domain by

$$
\psi_{1-j}(t)=\sum_{k=1}^{K}\left(\mathbb{C}_{j, k} * \psi_{k}\right)(t), \quad 1 \leq j \leq K .
$$

Relation (16) gives the exact boundary condition on the left boundary. Similarly, the exact boundary condition on the right writes

$$
\psi_{J+j}(t)=\sum_{k=1}^{K}\left(\mathbb{C}_{j, k} * \psi_{J-k+1}\right)(t), \quad 1 \leq j \leq K .
$$

Our goal is now to determine the kernel functions $\mathbb{C}_{j, k}(t)$. From $\mathbb{B}(t)=(\mathbb{C} * \mathbb{A})(t), \mathbb{A}_{j, k}(t)=$ $f_{j-k}(t)$ and $\mathbb{B}_{j, k}(t)=f_{j+k-1}(t)$, we remark that we only need to compute $f_{j}(t)$. Let us introduce the discrete Fourier series $U(s, x)$

$$
U(s, x)=\sum_{j=-\infty}^{\infty} e^{\mathrm{i} j x} \tilde{f}_{j}(s),
$$

where $\tilde{f}_{j}(s)$ denotes the Laplace transform of $f_{j}(t)$. From 10$)$, we obtain

$$
\begin{array}{r}
\mathrm{i} s(U-1)=a_{0}(U-1)+\sum_{k=1}^{K} a_{k}\left(\sum_{\ell=-\infty}^{\infty} e^{\mathrm{i} \ell x} \tilde{f}_{\ell+k}+\sum_{\ell=-\infty}^{\infty} e^{\mathrm{i} \ell x} \tilde{f}_{\ell-k}\right) \\
=a_{0}(U-1)+\sum_{k=1}^{K} a_{k}\left(e^{-\mathrm{i} k x} \sum_{\ell=-\infty}^{\infty} e^{\mathrm{i} \ell x} \tilde{f}_{\ell}+e^{\mathrm{i} k x} \sum_{\ell=-\infty}^{\infty} e^{\mathrm{i} \ell x} \tilde{f}_{\ell}\right) \\
=\left(a_{0}+2 \sum_{k=1}^{K} a_{k} \cos (k x)\right) U-a_{0}-\sum_{k=1}^{K} a_{k}\left(\tilde{f}_{k}+\tilde{f}_{-k}\right),
\end{array}
$$

which leads to

$$
U(s, x)=\frac{\mathrm{i} s-a_{0}-\sum_{k=1}^{K} a_{k}\left(\tilde{f}_{k}(s)+\tilde{f}_{-k}(s)\right)}{i s-a_{0}-2 \sum_{k=1}^{K} a_{k} \cos (k x)} .
$$

We also have the following relation

$$
\begin{aligned}
& 1=\frac{1}{2 \pi} \int_{0}^{2 \pi} U(s, x) d x \\
& =\left(\mathrm{i} s-a_{0}-\sum_{k=1}^{K} a_{k}\left(\tilde{f}_{k}(s)+\tilde{f}_{-k}(s)\right)\right) \frac{1}{2 \pi} \int_{0}^{2 \pi} \frac{1}{\mathrm{i} s-a_{0}-2 \sum_{k=1}^{K} a_{k} \cos (k x)} d x,
\end{aligned}
$$


which gives

$$
\frac{1}{\mathrm{i} s-a_{0}-\sum_{k=1}^{K} a_{k}\left(\tilde{f}_{k}(s)+\tilde{f}_{-k}(s)\right)}=\frac{1}{2 \pi} \int_{0}^{2 \pi} \frac{1}{\mathrm{i} s-a_{0}-2 \sum_{k=1}^{K} a_{k} \cos (k x)} d x .
$$

Now, we obtain

$$
\begin{aligned}
\tilde{f}_{j}(s) & =\frac{1}{2 \pi} \int_{0}^{2 \pi} U(s, x) e^{-\mathrm{i} j x} d x \\
& =\left(\frac{1}{2 \pi} \int_{0}^{2 \pi} \frac{e^{-\mathrm{i} j x}}{\mathrm{i} s-a_{0}-2 \sum_{k=1}^{K} a_{k} \cos (k x)} d x\right) /\left(\frac{1}{2 \pi} \int_{0}^{2 \pi} \frac{1}{\mathrm{i} s-a_{0}-2 \sum_{k=1}^{K} a_{k} \cos (k x)} d x\right) .
\end{aligned}
$$

By introducing

$$
\tilde{g}_{j}(s)=\frac{1}{2 \pi} \int_{-\pi}^{\pi} \frac{e^{-\mathrm{i} j x}}{\mathrm{i} s-a_{0}-2 \sum_{k=1}^{K} a_{k} \cos (k x)} d x
$$

we have

$$
\tilde{f}_{j}(s)=\frac{\tilde{g}_{j}(s)}{\tilde{g}_{0}(s)}
$$

Since $g_{j}(t)=g_{-j}(t)$, we only need to determine the functions $g_{j}(t)$, with $0 \leq j \leq 2 K-1$, to get $\mathbb{C}_{j, k}(t)$. We remark that $\widetilde{\mathbb{B}}=\widetilde{\mathbb{C}} \widetilde{\mathbb{A}}$, with $\widetilde{\mathbb{A}}_{j, k}(s)=\tilde{f}_{j-k}(s)=\tilde{g}_{j-k}(s) / \tilde{g}_{0}(s)$ and $\widetilde{\mathbb{B}}_{j, k}(s)=$ $\tilde{g}_{j+k-1}(s) / \tilde{g}_{0}(s)$. Therefore, we can modify the coefficients $\mathbb{A}_{j, k}(t)=g_{j-k}(t)$ and $\mathbb{B}_{j, k}(t)=g_{j+k-1}(t)$ such that $\mathbb{B}(t)=(\mathbb{C} * \mathbb{A})(t)$. It is obvious to see that, for any integer $j$, we have

$$
i s \tilde{g}_{j}-\delta_{j}^{0}=a_{0} \tilde{g}_{j}+\sum_{k=1}^{K} a_{k}\left(\tilde{g}_{j+k}+\tilde{g}_{j-k}\right),
$$

which leads to

$$
\begin{aligned}
& \mathrm{i} \dot{g}_{j}(t)=a_{0} g_{j}+\sum_{k=1}^{K} a_{k}\left(g_{j+k}+g_{j-k}\right), \\
& g_{j}(0)=-\mathrm{i} \delta_{j}^{0},
\end{aligned}
$$

where $\delta_{j}^{0}$ is the Krönecker delta symbol which is equal to 1 if $j=0$, and zero otherwise. On the other hand, we have the following equalities

$$
\begin{aligned}
& \frac{d}{d s} \sum_{k=1}^{K} k a_{k}\left(\tilde{g}_{j-k}-\tilde{g}_{j+k}\right)=\frac{d}{d s}\left(\frac{1}{2 \pi} \int_{-\pi}^{\pi} \frac{2 \mathrm{i} \sum_{k=1}^{K} k a_{k} \sin (k x) e^{-\mathrm{i} j x}}{\mathrm{i} s-a_{0}-2 \sum_{k=1}^{K} a_{k} \cos (k x)} d x\right) \\
& =\frac{1}{2 \pi} \int_{-\pi}^{\pi} \frac{2 \sum_{k=1}^{K} k a_{k} \sin (k x) e^{-\mathrm{i} j x}}{\left(\mathrm{i} s-a_{0}-2 \sum_{k=1}^{K} k a_{k} \cos (k x)\right)^{2}} d x \\
& =-\frac{1}{2 \pi} \int_{-\pi}^{\pi} \frac{e^{-\mathrm{i} j x}}{\left(\mathrm{i} s-a_{0}-2 \sum_{k=1}^{K} a_{k} \cos (k x)\right)^{2}} d\left(\mathrm{i} s-a_{0}-2 \sum_{k=1}^{K} a_{k} \cos (k x)\right) \\
& =\frac{\mathrm{i} j}{2 \pi} \int_{-\pi}^{\pi} \frac{e^{-\mathrm{i} j x}}{\mathrm{i} s-a_{0}-2 \sum_{k=1}^{K} a_{k} \cos (k x)} d x=\mathrm{i} j \tilde{g}_{j},
\end{aligned}
$$


which means that

$$
\sum_{k=1}^{K} k a_{k}\left(g_{j+k}(t)-g_{j-k}(t)\right)=\frac{\mathrm{i} j}{t} g_{j}(t)
$$

We can rewrite the above expression as

$$
g_{j}(t)=g_{j-2 K}(t)-\sum_{k=1}^{K-1} \frac{k a_{k}}{K a_{K}}\left(g_{j-K+k}(t)-g_{j-K-k}(t)\right)+\frac{\mathrm{i}(j-K)}{K a_{K} t} g_{j-K}(t),
$$

providing a recursive relation between the functions $g_{j}(t)$, that can also be computed through (29), with $0 \leq j \leq 2 K-1$. The left boundary $g_{j}(t)$, with $-K \leq j \leq 0$, can be calculated by $g_{j}(t)=g_{-j}(t)$. The right boundary $g_{j}(t)$, with $2 K \leq j \leq 3 K-1$, can be evaluated through (36). Therefore, we determine the ordinary system for $g_{j}(t)$, with $0 \leq j \leq 2 K-1$, and we obtain $\mathbb{C}_{j, k}$ from $\mathbb{B}=\mathbb{C} * \mathbb{A}$.

Let us remark that one of the strengths of our approach is that it can a priori be extended to higher dimensional problems. We refer e.g. to [38] for a similar approach where artificial boundary conditions are built for the local 2D Schrödinger equation. This approach can be adapted to the nonlocal context. In addition, all the developments also apply to nonlocal heat equations through some slight modifications.

\section{Full discretization scheme and implementation aspects}

In this Section, we develop the corresponding discretization of the reduced ordinary differential problem with the accurate artificial boundary condition. We adopt the Crank-Nicolson (CN) scheme with uniform time step $\Delta t>0$ for the time integration in (29)

$$
\left(1-\frac{a_{0} \Delta t}{2 \mathrm{i}}\right) g_{j}^{n+1}-\sum_{k=1}^{K} \frac{a_{k} \Delta t}{2 \mathrm{i}}\left(g_{j-k}^{n+1}+g_{j+k}^{n+1}\right)=\left(1+\frac{a_{0} \Delta t}{2 \mathrm{i}}\right) g_{j}^{n}+\sum_{k=1}^{K} \frac{a_{j} \Delta t}{2 \mathrm{i}}\left(g_{j-k}^{n}+g_{j+k}^{n}\right),
$$

with $g_{j}^{0}=-i \delta_{j}^{0}$ and $g_{j}^{n}=g_{-j}^{n}$. The notation $g_{j}^{n}$ corresponds to the approximation of $g_{j}$ at time $t_{n}=n \Delta t$, for $0 \leq n \leq N$, setting $\Delta t=T / N$. For the computation of $g_{j}$, with $0 \leq j \leq 2 K-1$, we need to evaluate $g_{j}$ for $2 K \leq j \leq 3 K-1$ and $-K \leq j \leq 1$ to close (37). We rewrite (36) with $j=2 K$ as

$$
g_{2 K}(t)=g_{0}(t)-\sum_{k=1}^{K-1} \frac{k a_{k}}{K a_{K}}\left(g_{K+k}(t)-g_{K-k}(t)\right)+\frac{\mathrm{i}}{a_{K} t} g_{K}(t)=\sum_{k=1}^{2 K} d_{k}^{1}(t) g_{2 K-k}(t) .
$$

Similarly, we have

$$
\begin{array}{r}
g_{2 K+1}(t)=\sum_{k=1}^{2 K} d_{k}^{1}(t) g_{2 K+1-k}(t)=d_{1}^{1}(t) g_{2 K}(t)+\sum_{k=2}^{2 K} d_{k}^{1}(t) g_{2 K+1-k}(t) \\
=d_{1}^{1}(t)\left(\sum_{k=1}^{2 K} d_{k}^{1}(t) g_{2 K-k}(t)\right)+\sum_{k=2}^{2 K} d_{k}^{1}(t) g_{2 K+1-k}(t)=\sum_{k=1}^{2 K} d_{k}^{2}(t) g_{2 K-k}(t) .
\end{array}
$$

Repeating the same procedure, for $1 \leq \ell \leq K$, we have

$$
g_{2 K+\ell-1}(t)=\sum_{k=1}^{2 K} d_{k}^{\ell}(t) g_{2 K-k}(t)
$$


where $d_{k}^{\ell}(t)$ is built by a recursive process that can be implemented numerically. Indeed, we have the following sequence of equalities

$$
\begin{aligned}
g_{2 K+\ell-1}(t)=\sum_{k=1}^{2 K} d_{k}^{1}(t) g_{2 K+\ell-1-k}(t)=\sum_{k=1}^{\ell-1} d_{k}^{1}(t) g_{2 K+\ell-1-k}(t)+\sum_{k=\ell}^{2 K} d_{k}^{1}(t) g_{2 K+\ell-1-k}(t) \\
=\sum_{k=1}^{\ell-1} d_{k}^{1}(t) \sum_{p=1}^{2 K} d_{p}^{\ell-k} g_{2 K-p}(t)+\sum_{k=\ell}^{2 K} d_{k}^{1}(t) g_{2 K+\ell-1-k}(t) \\
=\sum_{k=1}^{2 K}\left(\sum_{p=1}^{\ell-1} d_{k}^{\ell-p}(t) d_{p}^{1}(t)\right) g_{2 K-k}(t)+\sum_{k=1}^{2 K+1-\ell} d_{k+1-\ell}^{1}(t) g_{2 K-k}(t) \\
=\sum_{k=1}^{2 K+1-\ell}\left(d_{k+1-\ell}^{1}(t)+\sum_{p=1}^{\ell-1} d_{k}^{\ell-p}(t) d_{p}^{1}(t)\right) g_{2 K-k}(t) \\
+\sum_{k=2 K+2-\ell}^{\ell K}\left(\sum_{p=1}^{\ell-1} d_{k}^{\ell-p}(t) d_{p}^{1}(t)\right) g_{2 K-k}(t),
\end{aligned}
$$

which means by identification with (41) that we have

$$
d_{k}^{\ell}(t)=d_{k+1-\ell}^{1}(t)+\sum_{p=1}^{\ell-1} d_{k}^{\ell-p}(t) d_{p}^{1}(t)
$$

for $1 \leq k \leq 2 K+1-\ell$ and

$$
d_{k}^{\ell}(t)=\sum_{p=1}^{\ell-1} d_{k}^{\ell-p}(t) d_{p}^{1}(t)
$$

for $2 K+2-\ell \leq k \leq 2 K$, with

$$
d_{k}^{1}=\frac{k a_{k}}{K a_{K}}, \text { for } 1 \leq k \leq K-1,
$$

and

$$
d_{K}^{1}=\frac{\mathrm{i}}{a_{K} t}, \text { and } d_{k}^{1}=-\frac{k a_{k}}{K a_{K}}, \text { for } K+1 \leq k \leq 2 K .
$$

Thus, for $1 \leq \ell \leq K$, the adapted $\mathrm{CN}$ scheme related to (41) is

$$
\frac{\left(g_{2 K+\ell-1}^{n}+g_{2 K+\ell-1}^{n-1}\right)}{2}=\frac{1}{2}\left(\sum_{k=1}^{2 K}\left(d_{k}^{\ell}\right)^{n} g_{2 K-k}^{n}+\sum_{k=1}^{2 K}\left(d_{k}^{\ell}\right)^{n-1} g_{2 K-k}^{n-1}\right),
$$

which is equivalent to

$$
g_{2 K+\ell-1}^{n}=-g_{2 K+\ell-1}^{n-1}+\sum_{k=1}^{2 K}\left(d_{k}^{\ell}\right)^{n} g_{2 K-k}^{n}+\sum_{k=1}^{2 K}\left(d_{k}^{\ell}\right)^{n-1} g_{2 K-k}^{n-1} .
$$

The expression (44) allows to compute the functions $g_{j}$, for $2 K \leq j \leq 3 K-1$. In addition, from $g_{j}(t)=g_{-j}(t)$, we deduce that

$$
g_{j}^{n}=g_{-j}^{n},
$$

which determines the numerical values of $g_{j}$, for $-K \leq j \leq-1$. Equations (44)-(45) together with (37) close the computation of $g_{j}^{n}(t)$, for $0 \leq j \leq 2 K-1$. 
After the approximation of $g_{j}$ at $t_{n}$ by $g_{j}^{n}$, for $0 \leq j \leq 2 K-1$, we can determine $\mathbb{C}$ from $\mathbb{B}=\mathbb{C} * \mathbb{A}$, with $\mathbb{A}_{j, k}=g_{j-k}$ and $\mathbb{B}_{j, k}=g_{j+k-1}$. To this end, the convolutions are computed at times $t_{n}=n \Delta t$ through the trapezoidal rule to be consistent with the CN scheme as follows

$$
\mathbb{B}^{n}=\frac{\Delta t}{2} \mathbb{C}^{n} \mathbb{A}^{0}+\Delta t \sum_{m=1}^{n-1} \mathbb{C}^{m} \mathbb{A}^{n-m}+\frac{\Delta t}{2} \mathbb{C}^{0} \mathbb{A}^{n}
$$

where $\mathbb{A}^{m}$ approximates $\mathbb{A}$ at $t_{m}=m \Delta t$, which leads to

$$
\mathbb{C}^{n}=\left(\mathbb{B}^{n}-\Delta t \sum_{m=1}^{n-1} \mathbb{C}^{m} \mathbb{A}^{n-m}-\frac{\Delta t}{2} \mathbb{C}^{0} \mathbb{A}^{n}\right)\left(\mathbb{A}^{0} \Delta t / 2\right)^{-1},
$$

with $\mathbb{B}_{j, k}^{n}=g_{j+k-1}^{n}$ and $\mathbb{A}_{j, k}^{n}=g_{j-k}^{n}$, where the values of $g_{j}^{n}$ are provided by $(37)$.

To illustrate the scheme, let us consider for example the constant kernel function given by

$$
\gamma_{\eta}(\alpha, \beta)=\frac{2}{\eta^{3}},
$$

for $\alpha \in[-\eta, \eta]$. We use the expression (7) with $\eta=0.3$ and $\Delta x=1 / 20$ to construct the coefficients $a_{k}$ in (9). Let us define the $L^{\infty}$-error on $\mathbb{C}(t)$ at $t=T$ as

$$
e_{\infty}^{\mathbb{C}^{\Delta t}}:=\max _{1 \leq j, k \leq K}\left|\mathbb{C}_{j, k}^{\mathrm{ref}}(t=T)-\mathbb{C}_{j, k}^{\Delta t}(t=T)\right|,
$$

where $\mathbb{C}^{\mathrm{ref}}(t=T)$ is computed as a reference for a small value of $\Delta t=10^{-3}$ and the coefficients $\mathbb{C}_{j, k}^{\Delta t}(t=T)$ denote the numerical values $\mathbb{C}_{j, k}(t=T)$ with a time step $\Delta t$. We report the error $e_{\infty}^{\mathbb{C}^{\Delta t}}$ at time $T=2$ on Table 1 , showing that the second-order convergence is reached.

\begin{tabular}{|c||c|c|c|c|c|c|}
\hline$\Delta t$ & $10^{-2}$ & $5 \times 10^{-3}$ & $4 \times 10^{-3}$ & $2.5 \times 10^{-3}$ & $2 \times 10^{-3}$ & cvg. rate \\
\hline \hline$e_{\infty}^{\mathbb{C}^{\Delta t}}$ & $2.6 \times 10^{-1}$ & $6.7 \times 10^{-2}$ & $4.2 \times 10^{-2}$ & $1.7 \times 10^{-2}$ & $1.1 \times 10^{-2}$ & 1.97 \\
\hline
\end{tabular}

Table 1: $\quad L^{\infty}$-norm error $e_{\infty}^{\mathbb{C}^{\Delta t}}$ and convergence rate for computing $\mathbb{C}$ at time $T=2$.

Now, let us come back to the solution of $(9)$ with a compact initial data confined for the indices $1 \leq j \leq J$, based on the CN scheme. The left boundary condition of $\psi_{j}^{n}$, with $-(K-1) \leq j \leq 0$, and the right boundary condition of $\psi_{j}^{n}$, with $J+1 \leq j \leq J+K$, are needed to close the scheme. Now, we can determine the discrete boundary condition at the left point from (16) as

$$
\psi_{1-j}^{n}=\frac{\Delta t}{2} \sum_{k=1}^{K} \mathbb{C}_{j, k}^{0} \psi_{k}^{n}+\Delta t \sum_{k=1}^{K} \sum_{m=1}^{n-1} \mathbb{C}_{j, k}^{m} \psi_{k}^{n-m}+\frac{\Delta t}{2} \sum_{k=1}^{K} \mathbb{C}_{j, k}^{n} \psi_{k}^{0}, \quad 1 \leq j \leq K,
$$

and the right boundary scheme from (17) can be written as

$$
\psi_{J+j}^{n}=\frac{\Delta t}{2} \sum_{k=1}^{K} \mathbb{C}_{j, k}^{0} \psi_{J-k+1}^{n}+\Delta t \sum_{j=1}^{K} \sum_{m=1}^{n-1} \mathbb{C}_{j, k}^{m} \psi_{J-k+1}^{n-m}+\frac{\Delta t}{2} \sum_{k=1}^{K} \mathbb{C}_{j, k}^{n} \psi_{L-k+1}^{0}, \quad 1 \leq j \leq K .
$$

The boundary conditions (50) and (51) are combined with the interior Crank-Nicolson scheme (37) to solve the semi-discrete nonlocal Schrödinger equation. 


\section{Numerical examples}

\subsection{Example 1}

We consider the following first example for (2) setting $V(x)=0$ and for the constant kernel function given by

$$
\gamma(\alpha, \beta)=\frac{2}{\eta^{3}}
$$

with $\alpha \in[-\eta, \eta]$. The computational domain is chosen as $[-3,3]$ and the final time is $T=2$. The initial data is the gaussian

$$
\psi(x, 0)=5 e^{2 \mathrm{i} x-25 \frac{x^{2}}{4}} .
$$

First, we fix the spatial discretization step to $\Delta x=5 \times 10^{-2}$ and analyze the $L^{\infty}$-norm error $e_{\infty, T}^{\Delta x, \Delta t}$ defined by

$$
e_{\infty, T}^{\Delta x, \Delta t}:=\max _{1 \leq j \leq J}\left|\psi_{j}^{N}-\psi_{j}^{N, \text { ref }}\right|
$$

thanks to $\Delta t$, with $N \Delta t=T$. The reference solution $\psi^{n \text {,ref }}$ is computed for $\Delta x=5 \times 10^{-2}$ and $\Delta t=1 \times 10^{-3}$. For different values of $\eta$, we report the error $e_{\infty, T}^{\Delta x, \Delta t}$ at time $T=2$ in Table 2 . This example shows that the scheme is second-order in time as expected.

\begin{tabular}{|c||c|c|c|c|c|c|}
\hline$e_{\infty, T}^{\Delta x, \Delta t}$ & $\Delta t=10^{-2}$ & $\Delta t=5 \times 10^{-3}$ & $\Delta t=4 \times 10^{-3}$ & $\Delta t=2.5 \times 10^{-3}$ & $\Delta t=2 \times 10^{-3}$ & cvg. rate \\
\hline \hline$\eta=0.3$ & $5.05 \times 10^{-3}$ & $1.27 \times 10^{-3}$ & $8.16 \times 10^{-4}$ & $3.25 \times 10^{-4}$ & $2.12 \times 10^{-4}$ & 1.97 \\
\hline$\eta=0.5$ & $3.11 \times 10^{-2}$ & $7.76 \times 10^{-3}$ & $4.95 \times 10^{-3}$ & $1.91 \times 10^{-3}$ & $1.24 \times 10^{-3}$ & 2.01 \\
\hline$\eta=0.8$ & $5.72 \times 10^{-3}$ & $1.43 \times 10^{-3}$ & $9.17 \times 10^{-4}$ & $3.58 \times 10^{-4}$ & $2.34 \times 10^{-4}$ & 1.99 \\
\hline$\eta=1$ & $2.20 \times 10^{-3}$ & $5.51 \times 10^{-4}$ & $3.53 \times 10^{-4}$ & $1.38 \times 10^{-4}$ & $8.91 \times 10^{-5}$ & 1.99 \\
\hline
\end{tabular}

Table 2: Example 1: $L^{\infty}$-norm error $e_{\infty, T}^{\Delta x, \Delta t}\left(\Delta x=5 \times 10^{-2}\right)$ and convergence rate vs. $\Delta t$ for various values $\eta$.

Let us now fix the time step to $\Delta t=1 \times 10^{-3}$. The reference solution $\psi^{n, \text { ref }}$ is obtained for $\Delta x=2.5 \times 10^{-3}$ and $\Delta t=1 \times 10^{-3}$. We report in Table 3 the error $e_{\infty, T}^{\Delta x, \Delta t}$ at $T=2$ with respect to the mesh refinement $\Delta x$. For the various values of $\eta$, we observe that the convergence rate is equal to 2 according to $\Delta x$.

\begin{tabular}{|c||c|c|c|c|c|}
\hline$e_{\infty, T}^{\Delta x, \Delta t}$ & $\Delta x=1 / 20$ & $\Delta x=1 / 40$ & $\Delta x=1 / 50$ & $\Delta x=1 / 80$ & cvg. rate \\
\hline \hline$\eta=0.3$ & $7.36 \times 10^{-3}$ & $1.81 \times 10^{-3}$ & $1.13 \times 10^{-3}$ & $4.67 \times 10^{-4}$ & 1.99 \\
\hline$\eta=0.5$ & $4.74 \times 10^{-2}$ & $1.17 \times 10^{-2}$ & $7.42 \times 10^{-3}$ & $2.80 \times 10^{-3}$ & 2.03 \\
\hline$\eta=0.8$ & $2.41 \times 10^{-2}$ & $5.98 \times 10^{-3}$ & $3.80 \times 10^{-3}$ & $1.45 \times 10^{-3}$ & 2.03 \\
\hline$\eta=1$ & $1.39 \times 10^{-2}$ & $3.45 \times 10^{-3}$ & $2.19 \times 10^{-3}$ & $8.36 \times 10^{-4}$ & 2.03 \\
\hline
\end{tabular}

Table 3: Example 1: $L^{\infty}$-norm error $e_{\infty, T}^{\Delta x, \Delta t}\left(\Delta t=1 \times 10^{-3}\right)$ and convergence rate vs. $\Delta x$ for various values $\eta$.

Figure 1 (left) shows the evolution of the numerical solution in the bounded domain with artificial boundary conditions by setting $\Delta t=1 \times 10^{-3}$ and $\Delta x=1.25 \times 10^{-2}$, for the different values $\eta=0.3$, $0.5,0.8$ and 1 (top to bottom). We also report on Figure 1 (right) the pointwise error $\left|\psi_{j}^{n}-\psi_{j}^{n, \text { ref }}\right|$ (in $\log _{10}$ scale) between the computed solution $\psi^{n}($ in $[-3 ; 3] \times[0 ; 4])$ and a reference solution $\psi^{n \text {,ref }}$ computed on a very large spatial domain for $\Delta x=2.5 \times 10^{-3}$ and $\Delta t=1 \times 10^{-3}$ and then restricted 
to the smaller computational domain $[-3 ; 3] \times[0 ; 4]$. We observe that the behavior of the wave field is different according to the value of $\eta$. In addition, the spurious reflection at the boundary remains small and of the level as the numerical error of the interior scheme.

\subsection{Example 2}

The goal in this second example is to check that the method also provides a nonlocal discrete model which is consistent with local models by taking $\eta=0$. To this end, we consider the potential $V=0$ and solve the local Schrödinger equation $\mathrm{i} \psi+\partial_{x}^{2} \psi=0$ for the exact beam-like solution

$$
\psi^{\mathrm{ref}}(x, t)=\frac{1}{\sqrt{\zeta+\mathrm{i} t}} e^{\mathrm{i} k(x-k t)-\frac{(x-2 k t)^{2}}{4(\zeta+\mathrm{i} t)}}
$$

with $\zeta=0.04$ and wave number $k=2$. The spatial computational domain is $[-3,3]$ and the final time is $T=0.2$. We fix a very small time step $\Delta t=10^{-5}$ and vary the spatial discretization step $\Delta x$. We consider the three- and five-point stencil finite-difference schemes in space (see the two equations (8)). The $L^{\infty}$-norm error $e_{\infty, T}^{\Delta x, \Delta t}$ vs. $\Delta x$ is reported in Table 4 for both schemes. We clearly observe that the corresponding schemes are second- and fourth-order in space as expected. For completeness, we plot in Figure 2 the numerical solution (left) and the pointwise error (right, in $\log _{10}$ scale) in the domain according to the reference solution (55) for the fourth-order scheme. Here, we fix the computational domain as $[-3 ; 3]$, the final time is $T=2$ and the discretization scheme uses the values $\Delta x=3.125 \times 10^{-2}$ and $\Delta t=1 \times 10^{-4}$ for the fourth-order spatial discretization scheme. We observe that the error is of the order of $\mathcal{O}\left((\Delta x)^{4}+(\Delta t)^{2}\right)$ as expected.

\begin{tabular}{|c||c|c|c|c||c|}
\hline$\Delta x$ & $2^{-3}$ & $2^{-4}$ & $2^{-5}$ & $2^{-6}$ & cvg. rate \\
\hline \hline$e_{\infty, T}^{\Delta x, \Delta t}$ & $5.66 \times 10^{-1}$ & $1.37 \times 10^{-1}$ & $3.36 \times 10^{-2}$ & $8.44 \times 10^{-3}$ & 2.02 \\
\hline$e_{\infty, T}^{\Delta x, \Delta t}$ & $6.31 \times 10^{-2}$ & $3.95 \times 10^{-3}$ & $2.49 \times 10^{-4}$ & $1.68 \times 10^{-5}$ & 3.96 \\
\hline
\end{tabular}

Table 4: Example 2 : $L^{\infty}$-norm error $e_{\infty, T}^{\Delta x, \Delta t}\left(\Delta t=10^{-5}\right)$ and convergence rate vs. $\Delta x$, for the spatial second(second line) and fourth-order (third line) schemes at $T=0.2$.

\subsection{Example 3}

We consider in this last example the case of an inhomogeneous kernel function [27, 28]

$$
\gamma_{\eta}(\alpha, \beta)=\frac{40 \sqrt{10} \sigma}{\sqrt{\pi} \xi^{3}} e^{-\frac{10 \alpha^{2}}{\xi^{2}}}, \quad \text { for } \quad \alpha \in[-\eta, \eta]
$$

with $\xi=\eta(1+3 \operatorname{erfc}(\beta) / 4)$ and $\sigma=1+e^{-3 \beta^{2}}$. The computational domain is chosen as $[-4,4]$ and the final time of computation is $T=1$. The initial data is set as (55) and $V=0$. The reference solution is computed on the larger domain $[-15,15]$ to avoid any boundary effect, and for the discretization parameters $\Delta x=2.5 \times 10^{-3}$ and $\Delta t=2.5 \times 10^{-4}$. For $\eta=0.1$ and $\eta=0.5$, we report in Table 5 the $L^{\infty}$-norm error $e_{\infty, T}^{\Delta x, \Delta t}$ for various values of $\Delta x$, setting $\Delta t=2.5 \times 10^{-4}$. We observe again that the scheme is second-order in space. In addition, Fig. (3) shows the behaviour of the numerical solution (left) and the error ( $\log _{10}$ scale) in the domain (right). The reference solution is again computed in the larger domain $[-15,15]$ for the same values of $\Delta x$ and $\Delta t$. 

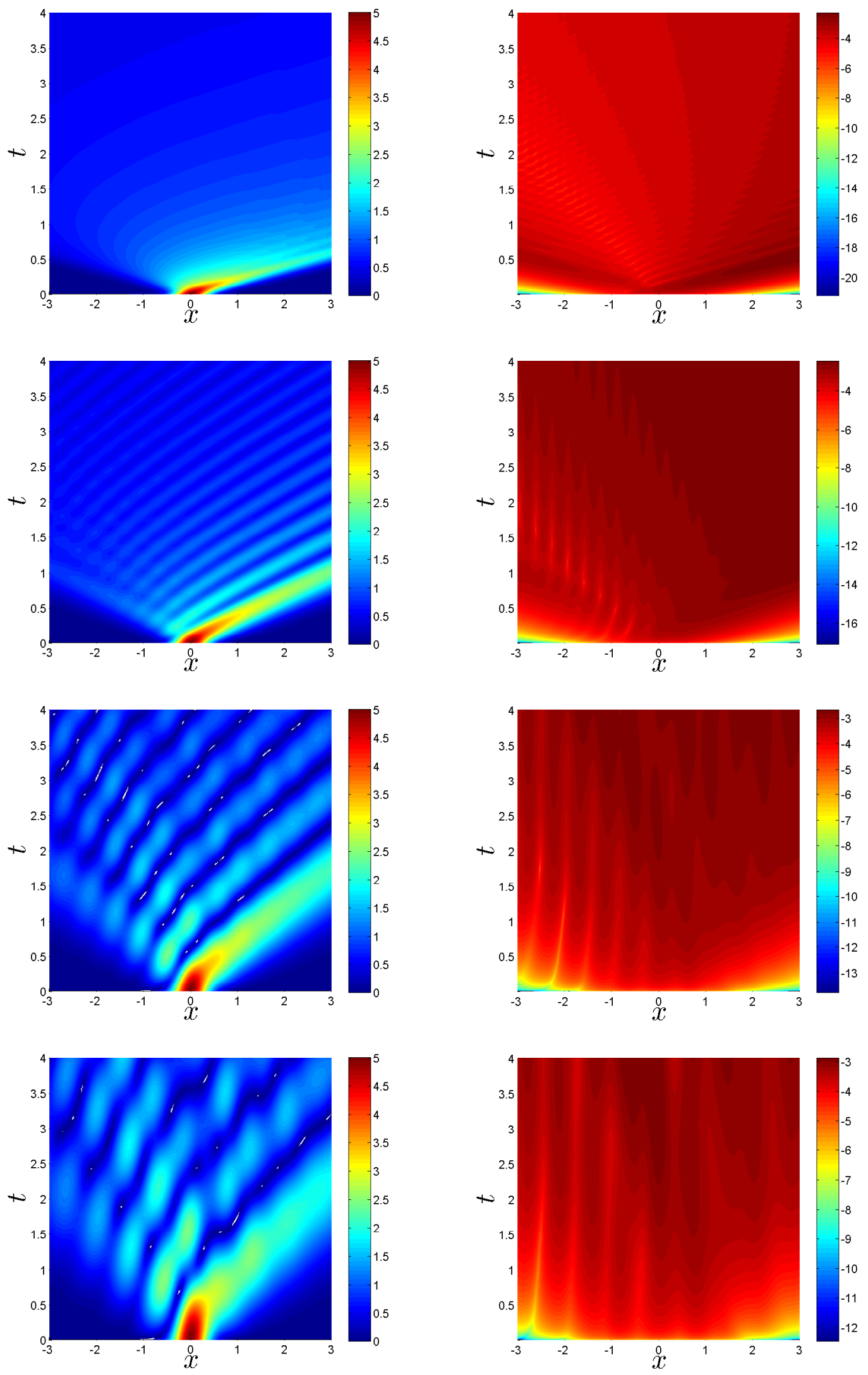

Figure 1: Example 1 (from top to bottom: $\eta=0.3,0.5,0.8,1$ ) : left: numerical solution; right: error between the reference and numerical solutions (in $\log _{10}$ scale). 

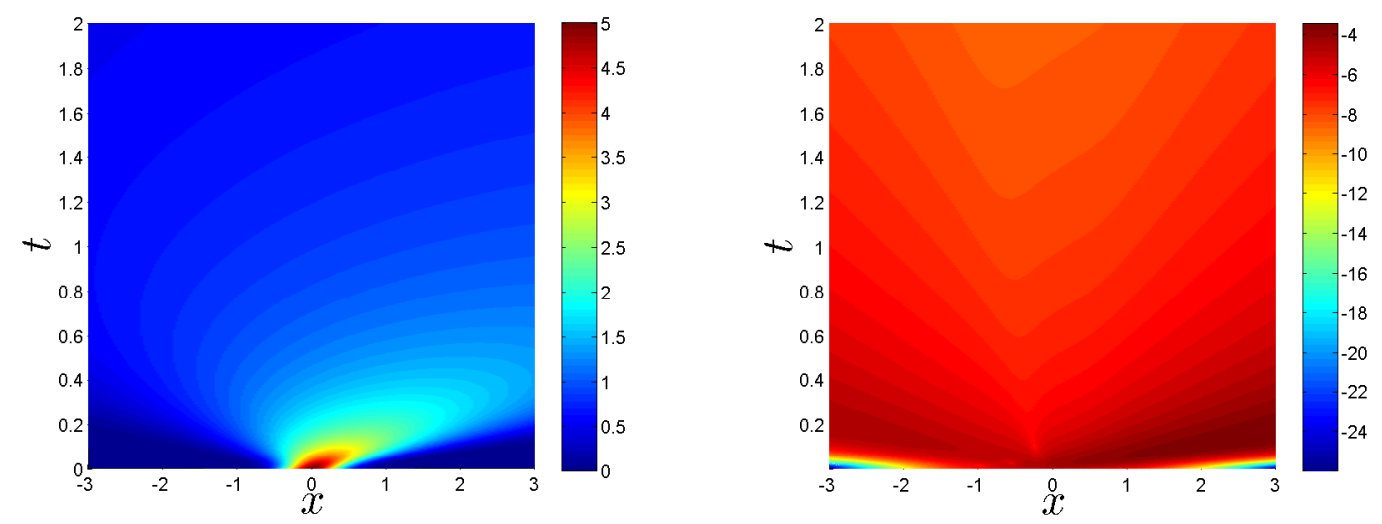

Figure 2: Example 2: left: numerical solution; right: error between the reference and numerical solutions (in $\log _{10}$ scale).

\begin{tabular}{|c||c|c|c|c||c|}
\hline$e_{\infty, T}^{\Delta x, \Delta t}$ & $\Delta x=5 \times 10^{-2}$ & $\Delta x=2 \times 10^{-3}$ & $\Delta x=1.25 \times 10^{-3}$ & $\Delta x=1 \times 10^{-3}$ & cvg. rate \\
\hline \hline$\eta=0.1$ & $1.51 \times 10^{-2}$ & $9.09 \times 10^{-3}$ & $3.09 \times 10^{-3}$ & $1.97 \times 10^{-3}$ & 2.24 \\
\hline$\eta=0.5$ & $2.49 \times 10^{-2}$ & $1.62 \times 10^{-2}$ & $7.23 \times 10^{-3}$ & $5.15 \times 10^{-3}$ & 1.72 \\
\hline
\end{tabular}

Table 5: Example $3(\eta=0.1,0.5): L^{\infty}$-norm error $e_{\infty, T}^{\Delta x, \Delta t}\left(\Delta t=2.5 \times 10^{-4}\right)$ and convergence rate vs. $\Delta x$ at $T=1$.

\section{Conclusion}

We proposed the construction of artificial boundary conditions for a one-dimensional nonlocal Schrödinger equation. To this end, we compute the kernel functions from an integral system by introducing some auxiliary functions $g_{j}(t)$. Fortunately, these functions can be obtained by solving an ordinary differential system by using the Crank-Nicolson scheme through recursive relations. The kernel functions are combined to build the exact transparent boundary conditions. These boundary conditions combined with the Crank-Nicolson scheme are used to solve the one-dimensional nonlocal Schrödinger equation. The second-order accuracy both in space and time of the resulting schemes is illustrated through three numerical examples.

The numerical algorithm presented here may be extended to any higher dimension and will be the topic of a future work. We further remark that the current approach may be applied to more general nonlocal PDEs. Regarding the artificial boundary conditions, some questions remain to prospect, in particular concerning the rigorous numerical analysis of the scheme (e.g. error estimates). In addition, the $\mathrm{CN}$ scheme that we developed requires the evaluation of convolutions which can be a limit to the approach. Both the acceleration of the calculations and improvement of the memory storage must then be reduced by suitable fast algorithms.

\section{Acknowledgments}

This research is partially supported by NSFC under grant Nos. NSFC 11832001, 11502028, 11771035, and the Natural Science Foundation of Hubei Province No. 2019CFA007 and Xiangtan University 2018ICIP01. This work also received the funding support of the ANR-17-CE40-0025 NABUCO project and the LIASFMA (Université de Lorraine). 

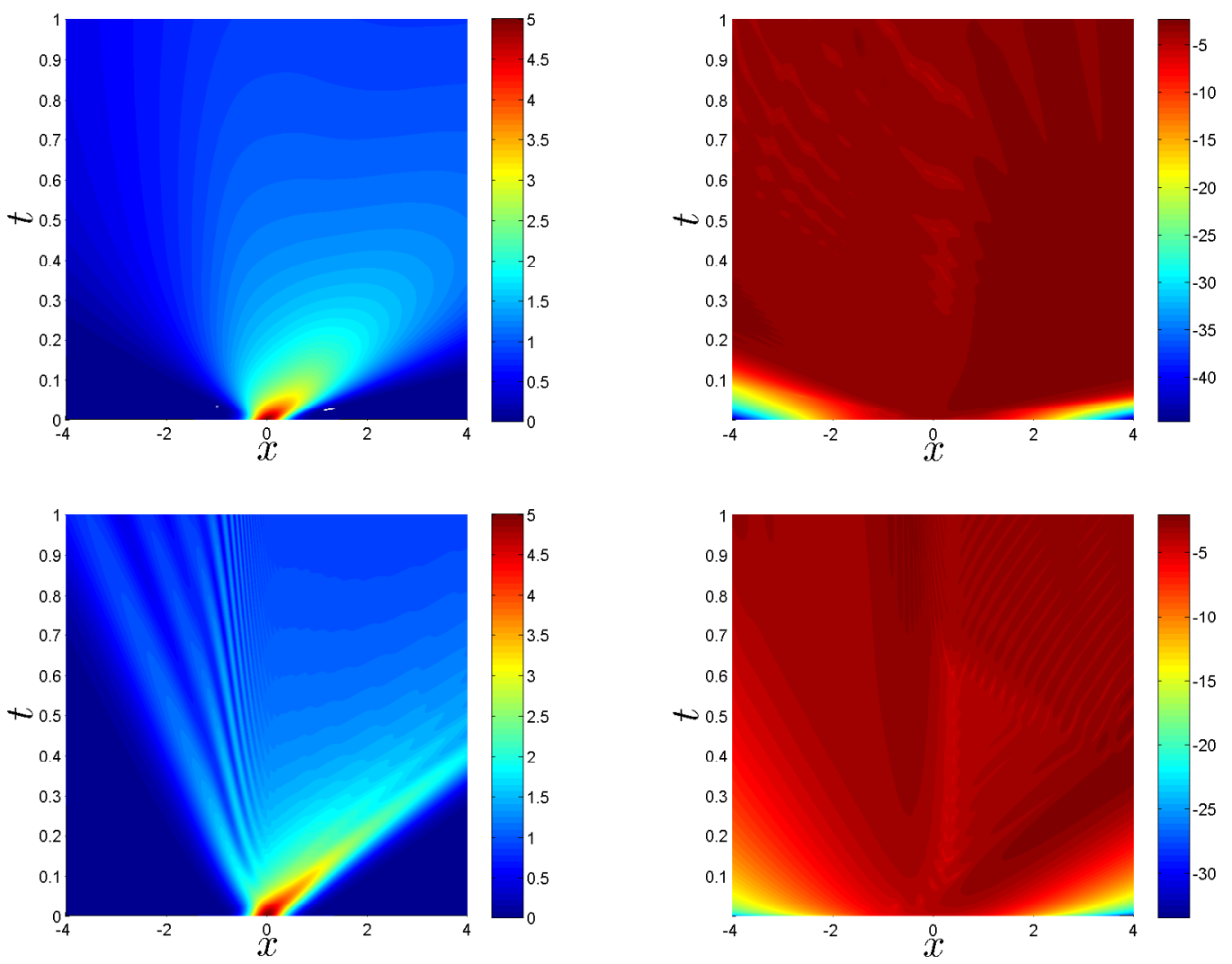

Figure 3: Example 3 (from top to bottom: $\eta=0.1,0.5$ ): left: numerical solution; right: error between the reference and numerical solutions (in $\log _{10}$ scale).

\section{References}

[1] J. Senior, Optical Fiber Communications: Principles and Practice, Prentice Hall, 1992.

[2] M. Levy, Parabolic Equation Methods for Electromagnetic Wave Propagation, IET, 2000.

[3] C. Sulem and P.L. Sulem, The Nonlinear Schrödinger Equation: Self-Focusing and Wave Collapse, Applied Mathematical Sciences 139, Springer, 1999.

[4] X. Antoine, W. Bao and C. Besse, Computational methods for the dynamics of the nonlinear Schrödinger/Gross-Pitaevskii equations, Computer Physics Communications 184 (12), (2013), pp.2621-2633.

[5] X. Antoine, A. Arnold, C. Besse, M. Ehrhardt, A. Schaedle, A review of transparent and artificial boundary conditions techniques for linear and nonlinear Schrödinger equations, Communications in Computational Physics 4, (2008), pp.729-796.

[6] X. Antoine, E. Lorin and Q. Tang, A friendly review of absorbing boundary conditions and perfectly matched layers for classical and relativistic quantum waves equations, Molecular Physics 115 (15-16), (2017), pp. 1861-1879.

[7] N. Laskin, Fractional quantum mechanics and Lévy path integrals, Physical Letter A 268 (2000), pp.298-305. 
[8] N. Laskin, Lévy flights over quantum paths, Communications in Nonlinear Science and Numerical Simulation 12, (2007), pp.2-18.

[9] N. Laskin, Fractals and quantum mechanics, Chaos 10, (2000), pp.780-790.

[10] N. Laskin, Fractional quantum mechanics, Physical Review E 62, (2000), pp.3135-3145.

[11] K. Kirkpatrick, E. Lenzmann, G. Staffilan, On the continuum limit for discrete NLS with longrange lattice interactions, Communications in Mathematical Physics 317, (2012), pp.563-591.

[12] S. Secchi, Ground state solutions for nonlinear fractional Schrödinger equations in $\mathbb{R}^{N}$, Journal of Mathematical Physics 54, (2013), 031501.

[13] X. Guo and M. Xu, Some physical applications of fractional Schrödinger equation, Journal of Mathematical Physics 47, (2015), 082104.

[14] X. Antoine, Q. Tang and Y. Zhang, On the ground states and dynamics of space fractional nonlinear Schrödinger/Gross-Pitaevskii equations with rotation term and nonlocal nonlinear interactions, Journal of Computational Physics, 325, (2016), pp.74-97.

[15] X. Antoine, Q. Tang and J. Zhang, On the numerical solution and dynamical laws of nonlinear fractional Schrödinger/Gross-Pitaevskii equations, International Journal of Computer Mathematics 95 (6-7), (2019), pp.1423-1443.

[16] M. Bhatti, Fractional Schrödinger wave equation and fractional uncertainty principle, International Journal of Contemporay Mathematical Sciences, 2 (2007), pp.943-950.

[17] A.H. Bhrawy and M.A. Abdelkawy, A fully spectral collocation approximation for multidimensional fractional Schrödinger equations, Journal of Computational Physics, 294, (2015), pp.462-483.

[18] A.H. Bhrawy and M.A. Zaky, Highly accurate numerical schemes for multi-dimensional space variable-order fractional Schrödinger equations, Computers and Mathematics with Applications, 73 (6), (2017), pp.1100-1117.

[19] A.Q.M. Khaliq, X. Liang and K.M. Furati, A fourth-order implicit-explicit scheme for the space fractional nonlinear Schrödinger equations, Numerical Algorithms, 75 (1), (2017), pp.147-172.

[20] K. Kirkpatrick and Y. Zhang, Fractional Schrödinger dynamics and decoherence, Physica D: Nonlinear Phenomena, 332, (2016), pp.41-54.

[21] X. Liang, A.Q.M. Khaliq, H. Bhatt and K.M. Furati, The locally extrapolated exponential splitting scheme for multi-dimensional nonlinear space-fractional Schrödinger equations, Numerical Algorithms, 76 (2017), pp.939-958.

[22] F. Pinsker, W. Bao, Y. Zhang, H. Ohadi, A. Dreismann and J. J. Baumberg, Fractional quantum mechanics in polariton condensates with velocity-dependent mass, Physical Review B, 92, (2015), 195310.

[23] A. Lischke, G. Pang, M. Gulian, F. Song, C. Glusa, X. Zheng, Z. Mao, W. Cai, M. Meerschaert, M. Ainsworth, and G. E. Karniadakis, What is the fractional laplacian? a comparative review with new results, Journal of Computational Physics, 404, (2020), 109009. 
[24] M.D'Elia, Q. Du, C. Glusz, M. Gunzburger, X. Tian and Z. Zhou, Numerical methods for nonlocal and fractional models, Acta Numerica, to appear (arXiv:2002.01401).

[25] C. Zheng, J. Hu, Q. Du, J. Zhang, Numerical solution of the nonlocal diffusion equation on the real line, SIAM Journal on Scientific Computing, 39, (2017), pp.1951-1968.

[26] W. Zhang, J. Yang, J. Zhang, and Q. Du, Absorbing boundary conditions for nonlocal heat equations on unbounded domain, Communications in Computational Physics, 21 (2017), pp.1639.

[27] C. Zheng, Q. Du, X. Ma and J. Zhang, Stability and error analysis for a second-order fast approximation of the local and nonlocal diffusion equations on the real line, SIAM Journal on Numerical Analysis, 58 (3), (2020), pp.1893-1917.

[28] Q. Du, J. Zhang, C. Zheng, Nonlocal wave propagation in unbounded multiscale media, Communications in Computational Physics, 24, (2018), pp.1049-1072.

[29] Q. Du, H. Han, J. Zhang and C. Zheng, Numerical solution of a two-dimensional nonlocal wave equation on unbounded domains, SIAM Journal on Scientific Computing, 40, (2018), pp.14301445 .

[30] Y. Yan, J. Zhang, and C. Zheng, Numerical computations of nonlocal Schrödinger equations on the real line, Communications on Applied Mathematics and Computation, 2, (2020), pp.241-260.

[31] J. Wang, J. Zhang and C. Zheng, Stability and error analysis for a second-order approximation of $1 D$ nonlocal Schrödinger equation under DtN-type boundary conditions, under review.

[32] B. Baeumer, M. Kovcs, M.M. Meerschaert, and H. Sankaranarayanan, Boundary conditions for fractional diffusion, Journal of Computational and Applied Mathematics, 336, (2018), pp.408424 .

[33] J.F. Kelly, H. Sankaranarayanan and M.M. Meerschaert, Boundary conditions for two-sided fractional diffusion, Journal of Computational Physics, 376, (2019), pp.1089-1107.

[34] X. Antoine and E. Lorin, Towards Perfectly Matched Layers for time-dependent space fractional PDEs, Journal of Computational Physics, 391, (2019), pp.59-90.

[35] X. Antoine, E. Lorin and Y. Zhang, Derivation and analysis of computational methods for fractional Laplacian equations with absorbing layers, Numerical Algorithms, to appear.

[36] G. Pang, S. Ji, Y. Yang, S. Tang, Eliminating corner effects in square lattice simulation, 62, (2018), pp.111-122.

[37] X. Wang, S. Tang, Matching boundary conditions for lattice dynamics, International Journal for Numerical Methods in Engineering, 93, (2013), pp.1255-1285.

[38] S. Ji, Y. Yang, G. Pang, X. Antoine, Accurate artificial boundary conditions for the semidiscretized linear Schrödinger and heat equations on rectangular domains, Computer Physics Communications, 222, (2018), pp.84-93.

[39] X. Tian, Q. Du, Analysis and comparison of different approximations to nonlocal diffusion and linear peridynamic equations, SIAM Journal on Numerical Analysis, 51, (2013), pp.3458-3482.

[40] X. Tian, Q. Du, Asymptotically compatible schemes and applications to robust discretization of nonlocal models, SIAM Journal on Numerical Analysis, 52, (2014), pp.1641-1665. 